\title{
Palinologia de sedimentos da turfeira do Banhado Amarelo, São Francisco de Paula, Rio Grande do Sul, Brasil. Fungos e criptógamas
}

\author{
Bianca Batista da Costa Spalding ${ }^{1,2}$ e Maria Luisa Lorscheitter ${ }^{1}$
}

Recebido: 15.10.2008; aceito: 19.03.2009

\begin{abstract}
Peat palynology from the Banhado Amarelo bog, São Francisco de Paula, Rio Grande do Sul, Brasil. Fungi and cryptogams). The vegetation analysis based on sediment palynology needs correct taxonomy of the palynomorphs. The aim of the research is to present reference material to this analysis in Quaternary sediments from the Brazilian Southern Plateau, using palynomorphs of fungi and cryptogams preserved in the peat from Banhado Amarelo bog $\left(29^{\circ} 19^{\prime} \mathrm{S}\right.$ and $50^{\circ} 08^{\prime}$ W), São Francisco de Paula, RS. The profile $(390 \mathrm{~cm})$ was collected with a Hiller Sampler. The chemical processing of the 30 extracted samples followed standard methodology, using $\mathrm{HCl}, \mathrm{HF}, \mathrm{KOH}$ and acetolysis. Light microscopy was used in the analysis. A radiocarbon dating at the base of the profile (26.080 $\pm 320 \mathrm{yrs}$ BP, Beta 245611) indicated the time interval. Palynomorphs from nine fungi, five algae, three bryophytes and 13 pteridophytes are presented. Descriptions, illustrations and, whenever possible, ecological data from the original organisms are included. The high number of taxa shows the potential of these palynomorphs to offers subsidies to the palaeoenvironment research of the last millennia from Southern Brazilian Plateau.
\end{abstract}

Key words: palynomorphs, taxonomy, Quaternary, Southern Brazil

RESUMO - (Palinologia de sedimentos da turfeira do Banhado Amarelo, São Francisco de Paula, Rio Grande do Sul, Brasil. Fungos e criptógamas). A análise da vegetação baseada em palinologia de sedimentos exige adequada taxonomia dos palinomorfos. O trabalho visa assim fornecer subsídios taxonômicos para esta análise em sedimentos quaternários do Planalto sul-brasileiro, através do estudo de palinomorfos de fungos e criptógamas preservados num perfil sedimentar da

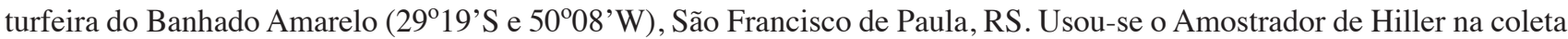
do perfil $(390 \mathrm{~cm})$. Dele extraíram-se 30 amostras, tratadas com $\mathrm{HCl}, \mathrm{HF}, \mathrm{KOH}$ e acetolisadas, com análise em microscopia óptica. Uma datação por ${ }^{14} \mathrm{C}$ na base da sequiência (26.080 \pm 320 anos AP, Beta 245611) estabeleceu o intervalo de tempo envolvido. Foram encontrados nove fungos, cinco algas, três briófitos e 13 pteridófitos. Incluem-se descrições, ilustrações e, sempre que possível, dados ecológicos do organismo de origem. A riqueza de táxons mostra a potencialidade também desses palinomorfos em oferecer subsídios ao conhecimento da história da vegetação dos últimos milênios do Planalto sul-brasileiro. Palavras-chave: palinomorfos, taxonomia, Quaternário, Sul do Brasil

\section{Introdução}

As mudanças climáticas ocorridas nos últimos milênios têm grande influência nos padrões fitogeográficos da atualidade por terem interferido na dinâmica da vegetação (Berglund 1986). Assim, o estudo da sucessão vegetal do Quaternário Tardio permite detectar processos envolvidos na gênese das formações vegetais atuais e a melhor compreensão da história e das tendências naturais dos ecossistemas. Pólen, esporos e outros palinomorfos preservados em sedimentos são uma importante ferramenta de trabalho na análise da história da vegetação por refletirem na sua morfologia a planta de origem e, consequentemente, seu ambiente. Por se preservarem em grande quantidade em ambientes deposicionais apropriados, como fundo de determinadas lagoas e pântanos, os palinomorfos de um perfil sedimentar permitem também análises quantitativas que tornam significativos os resultados obtidos. Quando a palinologia de sedimentos é associada a datações por ${ }^{14} \mathrm{C}$ o controle cronológico dos eventos pode ser estabelecido.

A palinologia de sedimentos para análise da vegetação pretérita exige uma correta identificação

1. Universidade Federal do Rio Grande do Sul, Instituto de Biociências, Departamento de Botânica, Av. Bento Gonçalves 9500,91540000 Porto Alegre, RS, Brasil

2. Autor para correspondência: biancaspalding@gmail.com 
dos palinomorfos preservados, porque dela depende fundamentalmente toda a reconstituição paleoambiental. Para tanto, trabalhos taxonômicos baseados na palinologia da flora atual e também de palinomorfos preservados em bacias deposicionais de uma região são de grande relevância como material básico de referência.

A dinâmica da mata com Araucária no Quaternário Tardio e a gênese das turfeiras do Planalto do Rio Grande do Sul tem despertado a atenção de muitos pesquisadores, mas ainda são poucos os trabalhos sobre palinologia de sedimentos na região, relacionados à análise de paleoambientes (Roth \& Lorscheitter 1993, Behling et al. 2001, 2004, Behling \& Pillar 2007, Leonhardt \& Lorscheitter 2007, Scherer \& Lorscheitter 2008, Roth \& Lorscheitter 2008). Visando contribuir para os estudos de sucessão vegetal no Planalto oriental do Sul do Brasil durante o Quaternário Tardio, é apresentada aqui a primeira parte da taxonomia de palinomorfos de um perfil sedimentar da turfeira do Banhado Amarelo, município de São Francisco de Paula, Rio Grande do Sul. Abrange a caracterização morfológica sucinta, taxonomia e, sempre que possível, dados ecológicos de palinomorfos ligados a fungos, algas, briófitos e pteridófitos, com fotomicrografias.

\section{Material e métodos}

A turfeira estudada integra o Banhado Amarelo $\left(29^{\circ} 19^{\prime}\right.$ S e $\left.50^{\circ} 08^{\prime} \mathrm{W}\right)$, município de São Francisco de Paula (figura 1). O perfil sedimentar foi coletado aproximadamente no centro da turfeira, correspondendo à porção mais espessa da bacia deposicional. Para a coleta do perfil foi usado o Amostrador de Hiller (Faegri \& Iversen 1989), em 15 secções de $26 \mathrm{~cm}$, que foram posteriormente alinhadas, formando um testemunho de $390 \mathrm{~cm}$ de comprimento. Ao longo desse perfil foram retiradas 30 amostras em intervalos regulares, cada uma contendo $8 \mathrm{~cm}^{3}$ de sedimento fresco. O processamento químico das amostras para análise palinológica seguiu o método padrão (Faegri \& Iversen 1989), usando-se $\mathrm{HCl}, \mathrm{HF}, \mathrm{KOH}$ e acetólise, com filtragem em malha de $250 \mu \mathrm{m}$. As lâminas para microscopia foram montadas em gelatina-glicerinada e a análise das mesmas foi realizada em fotomicroscópio óptico Diaplan Leitz em aumento de 400 vezes. A determinação do intervalo de tempo envolvido foi feita por uma datação por ${ }^{14} \mathrm{C}$ em sedimentos da base da seqüência, sendo realizada no laboratório Beta Analytic Inc., Miami, Flórida.
A palinoteca de plantas atuais do Laboratório de Palinologia do Departamento de Botânica da Universidade Federal do Rio Grande do Sul e diversos catálogos palinológicos serviram de base para a identificação do material. Devido à maior dificuldade taxonômica, para fungos e algas são indicadas no texto, sempre que possível, as referências que embasaram a identificação.

$\mathrm{Na}$ análise procurou-se sempre alcançar a categoria taxonômica mais inferior possível. Nos casos onde a morfologia dos esporos é muito semelhante dentro de um táxon, optou-se por usar a denominação "Tipo" antes do nome, conforme o usual em palinologia de sedimentos (Berglund 1986). Certos palinomorfos, que não puderam ser identificados, foram separados por números, possibilitando futura determinação taxonômica.

A ordenação taxonômica segue CABI (2008) para fungos, Round (1973) para algas, Bold et al. (1987) para briófitos e Tryon \& Tryon (1982) para pteridófitos. Em fungos e algas a nomenclatura utilizada baseou-se nas referências indicadas nas descrições taxonômicas e em briófitos e pteridófitos no glossário de Punt et al. (2007). Os palinomorfos foram descritos de modo sucinto, usando-se as características distintivas básicas para a identificação. As medidas foram realizadas em ocular de fio móvel e convertidas em micrômetros. Nas medidas dos esporos de briófitos e pteridófitos foram usados, sempre que possível, os eixos polar e equatorial. Fotomicrografias acompanham as descrições. A maioria dos palinomorfos foi fotografada em aumento de 1000 vezes (filme T-MAX 100). As descrições dos táxons são complementadas com dados ecológicos, fornecendo assim informações para análises de sucessão vegetal e de paleoambientes.

\section{Resultados}

A análise taxonômica revelou um total de 30 palinomorfos (nove fungos, cinco algas, três briófitos e treze pteridófitos) preservados ao longo do perfil sedimentar correspondente aos últimos 26.000 anos (amostra basal datada $26.080 \pm 320$ anos AP, Beta 245611).

Fungos

Filo Glomeromycota

Classe Glomeromycetes

Ordem Glomerales

Família Glomeraceae 

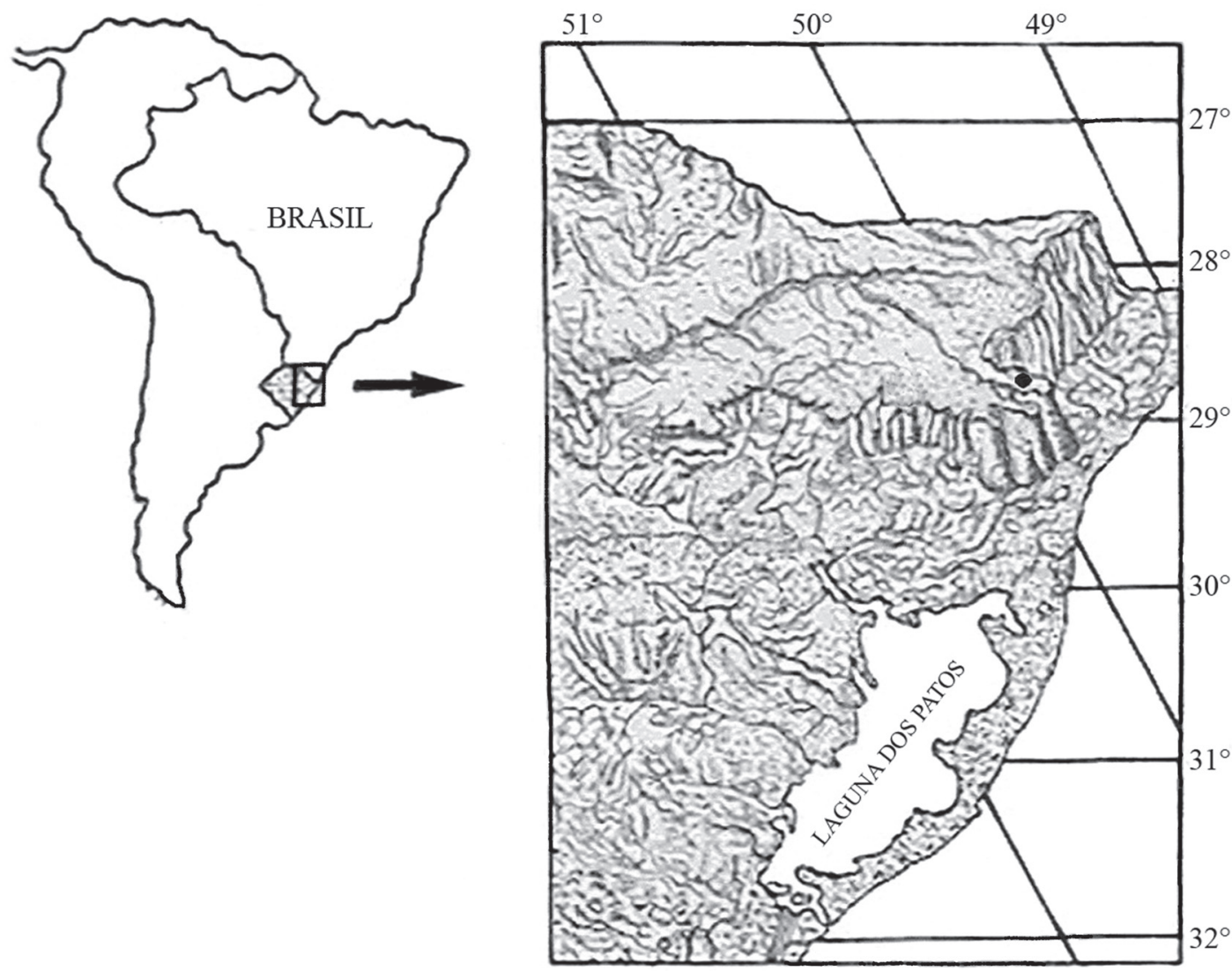

Figura 1. Estado do Rio Grande do Sul, Brasil e localização do perfil sedimentar estudado.

- = Banhado Amarelo (29¹9’S e 5008’W), São Francisco de Paula.

Figure 1. Rio Grande do Sul State, Brazil and the location of the sedimentary profile.

- = Banhado Amarelo (29 $19^{\prime}$ S and 50 08’ W), São Francisco de Paula.

1. Glomus Tul. \& C. Tul.

Figura 2

Clamidósporo amarelado, esferoidal, psilado e com parede celular engrossada. Sempre preso a filamento de hifa cenocítica. Diâmetro: 29-47 $\mu \mathrm{m}$.

Referências: Schüßler et al. (2001), Leal \& Lorscheitter (2006), Leonhardt \& Lorscheitter (2007), Scherer \& Lorscheitter (2008), Roth \& Lorscheitter (2008).

Citado para o Rio Grande do Sul pelos antigos nomes de Rhizophagites Rosendahl (Lorscheitter 1989) e Rhizophagus Dang (Neves \& Lorscheitter 1992, Neves \& Bauermann 2003).

Dados ecológicos: gênero mais comum entre os fungos micorrízicos arbusculares, ocorrendo como endossimbionte em raízes de muitas plantas vasculares (Schüßler et al. 2001).
Filo Ascomycota

Classe Leotiomycetes

Ordem Helotiales

Família Helotiaceae

2. Bryophytomyces sphagni (Navashin) Cif.

Figuras 3-5

Esporo hialino, esferoidal, frágil e psilado. Superfície com placas poligonais, dando ao grão um aspecto facetado. Diâmetro: cerca de $20 \mu \mathrm{m}$.

Referências: Ciferri (1953), Van Geel (1978), Matteri (1987), Roth \& Lorscheitter (2008).

Citado para o Rio Grande do Sul pelo basônimo Tilletia sphagni Navashin (Neves \& Lorscheitter 1992, Leonhardt \& Lorscheitter 2007). 
Dados ecológicos: parasita de esporângios de várias espécies de Sphagnum, inibindo a formação dos esporos deste musgo (Matteri 1987).

Filo Basidiomycota

Classe Agaricomycetes

Ordem Atheliales

Família Atheliaceae

\section{Tipo Athelia Pers.}

Figura 6

Agrupamento de células de cor marrom-escuro, aproximadamente elipsoidal. Células pequenas, numerosas, irregular e densamente dispostas. Diâmetro do agrupamento: eixo maior $42-70 \mu \mathrm{m}$, eixo menor 32-50 $\mu \mathrm{m}$.

Referências: Hooghiemstra (1984), Neves \& Bauermann (2003), Leal \& Lorscheitter (2006), Leonhardt \& Lorscheitter (2007), Scherer \& Lorscheitter (2008).

Dados ecológicos: o gênero é composto por organismos possivelmente patógenos de liquens e algas (Kirk et al. 2001).

Outros esporos

\section{Esporo 1}

Figura 7

Esporo hialino, fusiforme, psilado, com extremidades arredondadas e caracteristicamente mais espessadas. Eixo maior: 23-35 $\mu \mathrm{m}$. Eixo menor: 14-17 $\mu \mathrm{m}$

\section{Esporo 2}

Figura 8

Unidade de dispersão marrom-escuro, fusiforme, trisseptada, recoberta por um envoltório hialino com pequenas dobras irregulares por toda a superfície. Eixo maior: 28-44 $\mu \mathrm{m}$. Eixo menor: 16-20 $\mu \mathrm{m}$.

Referências: Neves \& Lorscheitter (1992), Scherer \& Lorscheitter (2008).

\section{Esporo 3}

Figura 9

Unidade de dispersão marrom-escuro, fusiforme, trisseptada, psilada. Eixo maior: 25-39 $\mu \mathrm{m}$. Eixo menor: $10-16 \mu \mathrm{m}$.

\section{Esporo 4}

Figura 10

Unidade de dispersão marrom-escuro, fusiforme, monosseptada, psilada. Eixo maior: cerca de $45 \mu \mathrm{m}$. Eixo menor: cerca de $12 \mu \mathrm{m}$.

\section{Esporo 5}

Figuras 11-12

Esporo marrom-escuro, esferoidal, pequeno, microequinado. Espinhos muito finos, densamente dispostos. Perfuração evidente, com espessamento marginal. Diâmetro: cerca de $10 \mu \mathrm{m}$.

Referência: Leonhardt \& Lorscheitter (2007).

\section{Esporo 6}

Figuras 13-14

Esporo marrom, esferoidal, tuberculado.Projeções irregularmente dispostas. Robusto. Diâmetro: 63 $\mu \mathrm{m}$.

\author{
Algas \\ Divisão Chlorophyta \\ Classe Chlorophyceae \\ Ordem Chlorococcales \\ Família Dictyosphaeriaceae
}

\section{Botryococcus Kützing}

Figuras 15-16

Colônia irregularmente lobada, de tamanho variável, psilada, formada por muitos indivíduos unicelulares, densa e concentricamente dispostos. Diâmetro da colônia: eixo maior 32-49 $\mu \mathrm{m}$, eixo menor 20-35 $\mu \mathrm{m}$.

Referências: Hooghiemstra (1984), Neves \& Bauermann (2003), Leonhardt \& Lorscheitter (2007), Scherer \& Lorscheitter (2008), Roth \& Lorscheitter (2008).

Dados ecológicos: em água doce de poças ou lagos (Erdtman 1969).

Classe Zygnemaphyceae

Ordem Zygnemales

Família Zygnemaceae

11. Debarya (De Bary) Wittrock

Figuras 17-18

Zigósporo hialino, formado por hemisférios complementares, radialmente simétricos. Cada 

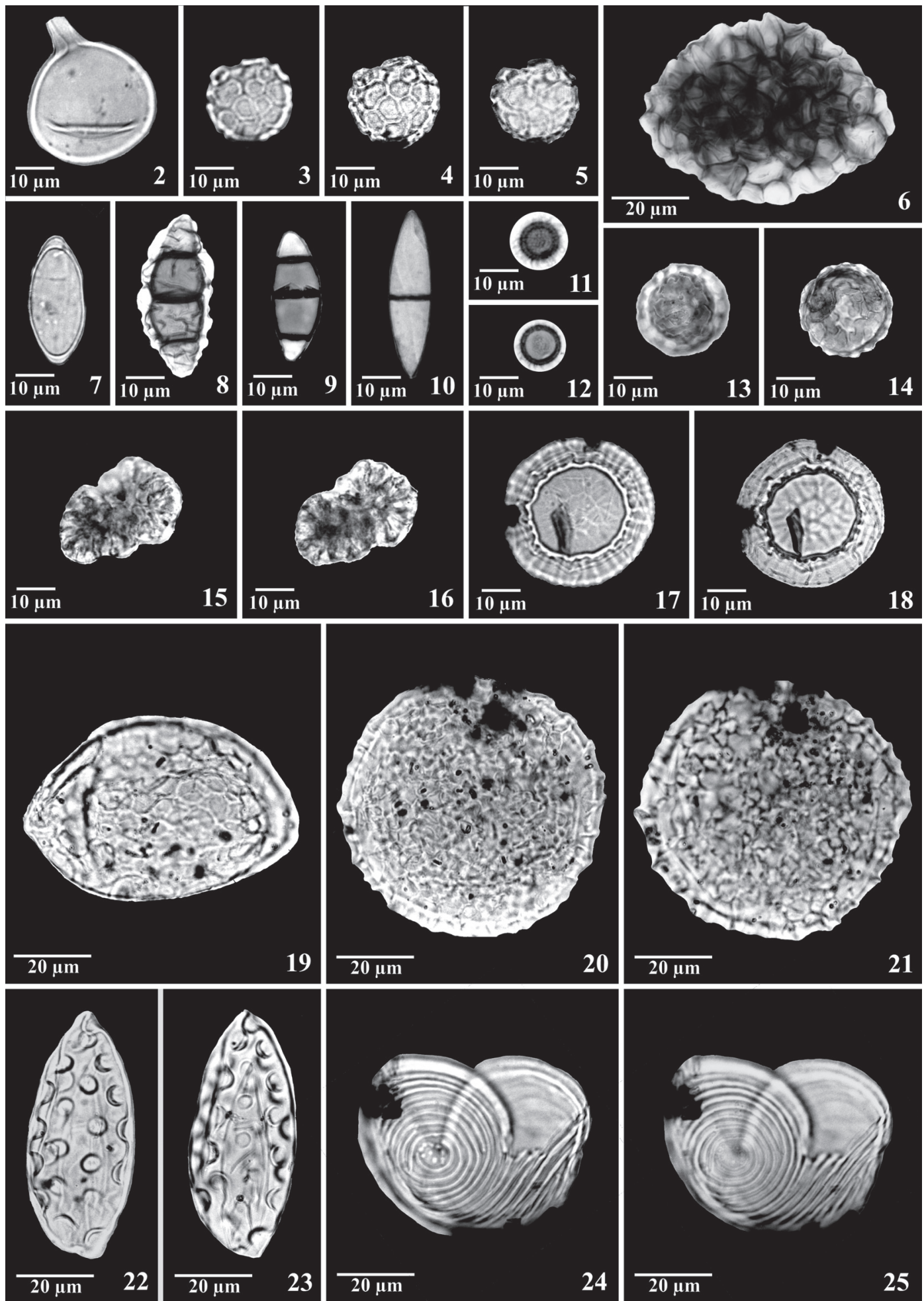

Figuras 2-14. Fungos. 2. Glomus. 3-5. Bryophytomyces sphagni: $1^{\circ}-3^{\circ}$ pl. 6. Tipo Athelia. 7. Esporo 1. 8. Esporo 2. 9. Esporo 3. 10. Esporo 4. 11-12. Esporo 5: $1^{\circ}-2^{\circ} \mathrm{pl} .13-14$. Esporo 6: $1^{\circ}-2^{\circ} \mathrm{pl}$. 15-25. Algas. 15-16. Botryococcus: $1^{\circ}-2^{\circ} \mathrm{pl} .17-18$. Debarya: $1^{\circ}-2^{\circ} \mathrm{pl} .19-21$. Spirogyra. 20-21: $1^{\circ}-2^{\circ} \mathrm{pl}$. 22-23. Zygnema: $1^{\circ}-2^{\circ} \mathrm{pl}$. 24-25. Pseudoschizaea rubina: $1^{\circ}-2^{\circ} \mathrm{pl}$. ( $\mathrm{pl}=$ planos de focalização).

Figures 2-14. Fungi. 2. Glomus. 3-5. Bryophytomyces sphagni: $1^{\circ}-3^{\circ}$ pl. 6. Athelia type. 7. Spore 1. 8. Spore 2. 9. Spore 3. 10. Spore 4. 11-12. Spore 5: $1^{\circ}-2^{\circ} \mathrm{pl}$. 13-14. Spore 6: $1^{\circ}-2^{\circ} \mathrm{pl}$. 15-25. Algae. 15-16. Botryococcus: $1^{\circ}-2^{\circ} \mathrm{pl}$. 17-18. Debarya: $1^{\circ}-2^{\circ} \mathrm{pl} .19-21$. Spirogyra . 20-21: $1^{\circ}-2^{\circ}$ pl. 22-23. Zygnema: $1^{\circ}-2^{\circ}$ pl. 24-25. Pseudoschizaea rubina: $1^{\circ}-2^{\circ} \mathrm{pl}$. (pl = planes $)$. 
hemisfério com espessamento anelar característico em vista frontal, com estrias finas e radialmente dispostas no centro, e outras, também de disposição radial, na periferia. Diâmetro equatorial: cerca de $38 \mu \mathrm{m}$.

Referências: Hooghiemstra (1984), Neves \& Lorscheitter (1992), Neves \& Bauermann (2003), Leal \& Lorscheitter (2006), Leonhardt \& Lorscheitter (2007), Scherer \& Lorscheitter (2008), Roth \& Lorscheitter (2008).

Encontrados apenas fragmentos correspondentes a um dos hemisférios.

Dados ecológicos: em água doce estagnada e de pouca profundidade (Van Geel \& Van Der Hammen 1977).

\section{Spirogyra Link}

Figuras 19-21

Zigósporo hialino, às vezes amarelado, esferoidal a elipsoidal, reticulado. Retículo grosseiro e irregular. Zigósporos elipsoidais: eixo maior: 64-87 $\mu \mathrm{m}$, eixo menor: 40-48 $\mu \mathrm{m}$. Zigósporos esferoidais: diâmetro com cerca de $70 \mu \mathrm{m}$.

Referências: Hooghiemstra (1984), Neves \& Lorscheitter (1992), Neves \& Bauermann (2003), Leal \& Lorscheitter (2006), Leonhardt \& Lorscheitter (2007), Scherer \& Lorscheitter (2008).

Dados ecológicos: em água doce e em solos úmidos (Joly 2002).

\section{Zygnema C. Agardh}

Figuras 22-23

Zigósporo hialino, esferoidal a elipsoidal, psilado, apresentando reentrâncias circulares características, pequenas ou robustas, com distribuição regular por toda a superfície. Eixo maior: 52-65 $\mu \mathrm{m}$. Eixo menor: 26-36 $\mu \mathrm{m}$.

Referências: Van Geel (1978), Hooghiemstra (1984), Neves \& Lorscheitter (1992), Neves \& Bauermann (2003), Leal \& Lorscheitter (2006), Leonhardt \& Lorscheitter (2007), Scherer \& Lorscheitter (2008), Roth \& Lorscheitter (2008).

Dados ecológicos: em água doce e solos úmidos (Smith 1987, Joly 2002), ocorrendo também em água estagnada e rasa (Van Geel \& Van Der Hammen 1977).

\section{Incertae sedis}

14. Pseudoschizaea rubina Rossignol ex Christopher

Figuras 24-25
Grão hialino, circular em vista frontal onde apresenta estrias finas e concêntricas, formando anéis e espiras às vezes incompletos. Diâmetro em vista frontal: $35-43 \mu \mathrm{m}$.

Referências: Christopher (1976), Lorscheitter (1989), Neves \& Lorscheitter (1992), Neves \& Bauermann (2003), Leal \& Lorscheitter (2006), Leonhardt \& Lorscheitter (2007), Scherer \& Lorscheitter (2008), Roth \& Lorscheitter (2008).

Citado em trabalhos antigos como a forma A de Concentricystes rubinus Rossignol (Rossignol 1962).

Dados ecológicos: palinomorfo sem afinidade botânica conhecida, possivelmente originado de algas dulciaqüícolas (Rossignol 1962). Christopher (1976) refere-se a esse material como de um grupo incerto dentro das algas.

Briófitos

Divisão Anthocerotophyta

Classe Anthocerotopsida

Ordem Anthocerotales

Família Anthocerotaceae

\section{Aspiromitus punctatus (L.) Schljakov}

Figuras 26-27

Radiossimétrico, heteropolar, circular a subtriangular em vista polar. Trilete, raios finos com extremidade bifurcada. Exospório reticulado-equinado no pólo distal, psilado no proximal. Retículo grosseiro e irregular, com espinhos alongados e frequientemente bifurcados a trifurcados, alguns simples, sobre o muro do retículo. Eixo equatorial: 66-72 $\mu \mathrm{m}$.

Citado para o Rio Grande do Sul pelo basônimo Anthoceros punctatus L. (Lorscheitter 1989, Neves \& Bauermann 2004, Leonhardt \& Lorscheitter 2007).

Dados ecológicos: cosmopolita, em relvas úmidas (Schultz 1980).

\section{Phaeoceros laevis (L.) Prosk.}

Figuras 28-29

Radiossimétrico, heteropolar, circular em vista polar. Trilete, raios com extremidade bifurcada. Exospório microequinado a psilado no pólo distal, psilado no proximal. Eixo equatorial: 75-99 $\mu \mathrm{m}$.

Dados ecológicos: sobre solos úmidos, em locais sombreados. Comum em margem de arroios, rios, vertentes e em campos úmidos (Menéndez 1962). 

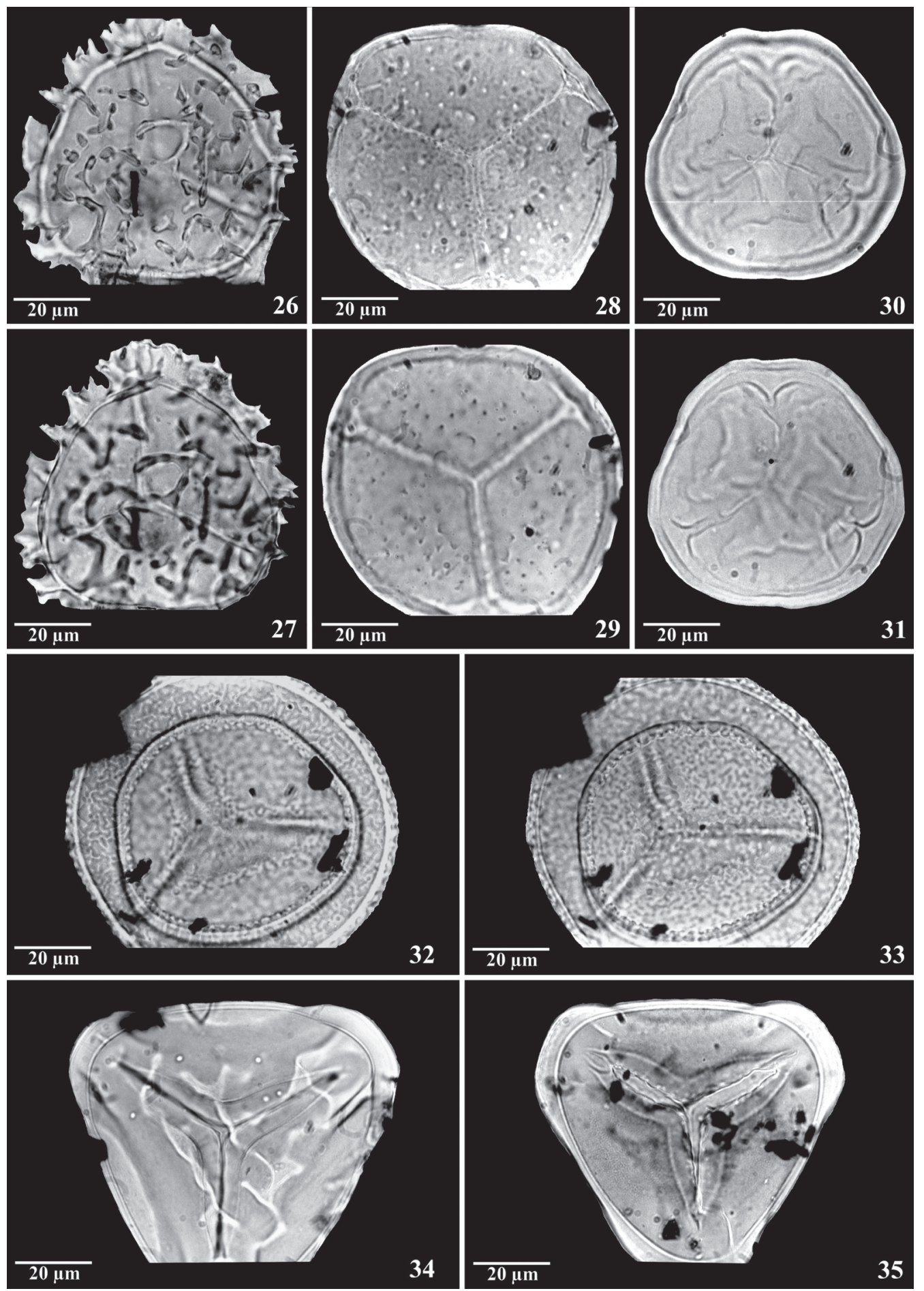

Figuras 26-31. Briófitos. 26-27. Aspiromitus punctatus (PD): $1^{\circ}-2^{\circ} \mathrm{pl}$ (marca trilete vista por transparência). 28-29. Phaeoceros laevis (PD): $1^{\circ}-2^{\circ} \mathrm{pl}$ (marca trilete vista por transparência). 30-31. Sphagnum cuspidatum var. recurvum (PP): $1^{\circ}-2^{\circ}$ pl. 32-35. Pteridófitos. 32-33. Osmunda (PD): $1^{\circ}-2^{\circ} \mathrm{pl}$ (marca trilete vista por transparência). 34-35. Dicksonia sellowiana. 34. (PD), (marca trilete vista por transparência), 35. (PP). ( $\mathrm{pl}=$ planos de focalização; $\mathrm{PD}=$ pólo distal; $\mathrm{PP}=$ pólo proximal).

Figures 26-31. Bryophytes. 26-27. Aspiromitus punctatus (PD): $1^{\circ}-2^{\circ} \mathrm{pl}$ (laesura seen due to transparency). 28-29. Phaeoceros laevis (PD): $1^{\circ}-2^{\circ} \mathrm{pl}$ (laesura seen due to transparency). 30-31. Sphagnum cuspidatum var. recurvum (PP): $1^{\circ}-2^{\circ}$ pl. 32-35. Pteridophytes. 32-33. Osmunda (PD): $1^{\circ}-2^{\circ} \mathrm{pl}$ (laesura seen due to transparency). 34-35. Dicksonia sellowiana. 34. (PD), (laesura seen due to transparency). 35. $(\mathrm{PP})$. $(\mathrm{pl}=$ planes $; \mathrm{PD}=$ distal pole $; \mathrm{PP}=$ proximal pole $)$. 
Divisão Bryophyta

Classe Sphagnopsida

Ordem Sphagnales

Família Sphagnaceae

17. Sphagnum cuspidatum var. recurvum (P. Beauv.) Wilson

Figuras 30-31

Radiossimétrico, heteropolar, subtriangular em vista polar, com zonas interangulares levemente convexas. Trilete. Exospório com característico espessamento trilobado no pólo distal, lobos irregularmente dispostos. Psilado no pólo proximal. Eixo equatorial: 49-69 $\mu \mathrm{m}$.

A espécie ocorre na atual turfeira, segundo a morfologia descrita por Yano et al. (1985).

Dados ecológicos: as espécies de Sphagnum ocorrem em solos ácidos, sujeitos a inundações, como brejos e pântanos, em especial turfeiras. Também em lagoas rasas, em densas e extensas colônias (Joly 2002).

Pteridófitos

\section{Divisão Pteridophyta \\ Classe Filicopsida \\ Ordem Polypodiales \\ Subordem Polypodiineae \\ Família Osmundaceae}

\section{Osmunda L.}

Figuras 32-33

Radiossimétrico, heteropolar, circular em vista polar. Trilete, raios longos e finos. Exospório fino, facilmente dobrável, tornando o grão frágil. Verrucado, verrugas de tamanho, forma e distribuição irregulares. Robusto. Eixo equatorial: cerca de $80 \mu \mathrm{m}$.

Esporo freqüentemente dobrado e fragmentado nas amostras.

Dados ecológicos: em ambientes úmidos, raro em terrenos com boa drenagem. Mais freqüente em locais abertos, mas também nos sombreados. Na América tropical geralmente ocorre em ambientes sempre úmidos, como pântanos, turfeiras, campos e margens de lagos (Tryon \& Tryon 1982). Comuns no Planalto do Rio Grande do Sul (Lorscheitter et al. 1998).

Família Dicksoniaceae

19. Dicksonia sellowiana Hook.

Figuras 34-35
Radiossimétrico, heteropolar, subtriangular a triangular em vista polar, zonas interangulares quase retas, ângulos mais espessados e truncados. Trilete, margo desenvolvido. Exospório com projeções largas e irregulares no pólo distal, psilado no proximal. Robusto. Eixo equatorial: 76-91 $\mu \mathrm{m}$.

Dados ecológicos: arborescente, geralmente em matas úmidas de montanha e florestas nebulares na América tropical, na base de encostas ou ao longo de córregos (Tryon \& Tryon 1982). No Rio Grande do Sul é encontrada em altitudes de 60 a $1200 \mathrm{~m}$, sendo freqüente na Mata com Araucária (Lorscheitter et al. 1999).

Família Lophosoriaceae

20. Lophosoria quadripinnata (J.F. Gmelin) Christensen

Figuras 36-37

Radiossimétrico, heteropolar, circular a subtriangular em vista polar. Zona equatorial fortemente expandida (cingulum). Trilete, raios proeminentes e verrucados. Exospório grosseiramente tuberculado e com algumas perfurações muito finas no pólo proximal, entre os raios. Pólo distal com várias perfurações também muito finas (punctado). Eixo equatorial: cerca de $80 \mu \mathrm{m}$.

Primeira citação da espécie para o Quaternário do Rio Grande do Sul.

Dados ecológicos: em ambientes úmidos e parcialmente sombreados, como barrancos e margens de formações florestais, desde as encostas de montanhas costeiras até $1000 \mathrm{~m}$ de altitude (Lorscheitter et al. 1999).

Família Cyatheaceae

21. Cyatheaceae

Figuras 38-39

Radiossimétrico, heteropolar, subtriangular a triangular em vista polar, zonas interangulares retas a levemente côncavas. Trilete, raios muito longos e finos. Exospório psilado, com espessamento uniforme. Eixo equatorial: $48-65 \mu \mathrm{m}$.

Dados ecológicos: em florestas tropicais de montanha, florestas úmidas de montanha ou em florestas nebulares. Também ao longo de rios em locais rochosos (Tryon \& Tryon 1982, Lorscheitter et al. 1999).

Família Pteridaceae 

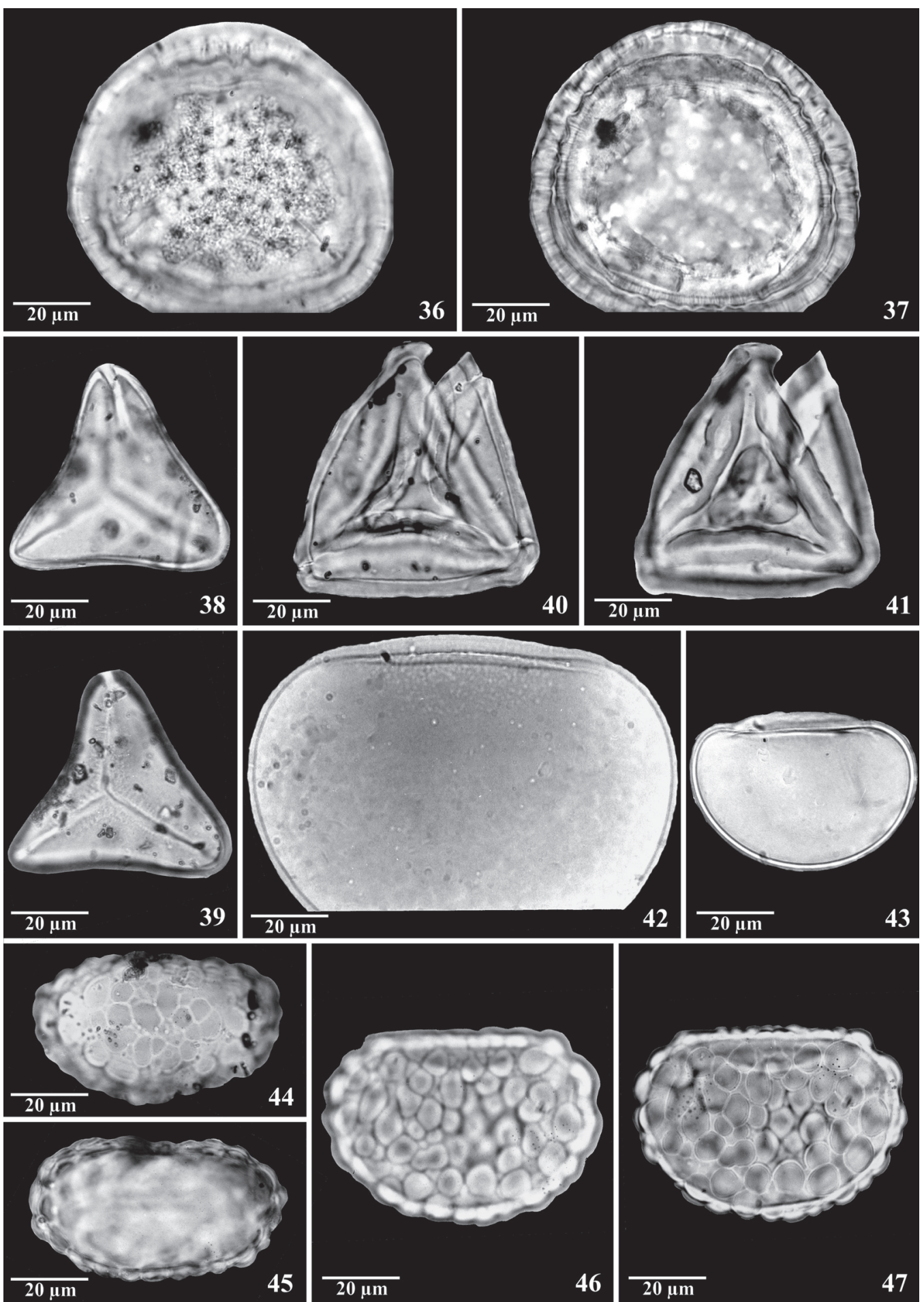

Figuras 36-47. Pteridófitos. 36-37. Lophosoria quadripinnata (PD): $1^{\circ}-2^{\circ} \mathrm{pl}$. 38-39. Cyatheaceae (PD): $1^{\circ}-2^{\circ} \mathrm{pl}$ (marca trilete vista por transparência). 40-41. Tipo Anogramma (PP): $1^{\circ}-2^{\circ} \mathrm{pl}$ (fraturado). 42. Blechnum cf. imperiale (EQ). 43. Tipo Blechnum (EQ). $44-47$. Pecluma pectinatiformis. 44-45. (PD): $1^{\circ}-2^{\circ} \mathrm{pl}, 46-47$. EQ: $1^{\circ}-2^{\circ} \mathrm{pl}$. ( $\mathrm{pl}=$ planos de focalização; $\mathrm{PD}=$ pólo distal; $\mathrm{PP}=$ pólo proximal; $\mathrm{EQ}=$ vista equatorial).

Figures 36-47. Pteridophytes. 36-37. Lophosoria quadripinnata (PD): $1^{\circ}-2^{\circ} \mathrm{pl} .38-39$. Cyatheaceae (PD): $1^{\circ}-2^{\circ} \mathrm{pl}$ (laesura seen due to transparency). 40-41. Anogramma type (PP): $1^{\circ}-2^{\circ} \mathrm{pl}$ (fractured). 42. Blechnum cf. imperiale (EQ). 43. Blechnum type (EQ). 44-47. Pecluma pectinatiformis. 44-45. (PD): $1^{\circ}-2^{\circ} \mathrm{pl}, 46-47 . \mathrm{EQ}: 1^{\circ}-2^{\circ} \mathrm{pl} .(\mathrm{pl}=$ planes; $\mathrm{PD}=$ distal pole; $\mathrm{PP}=$ proximal pole; $\mathrm{EQ}=\mathrm{equatorial}$ view $)$. 


\section{Tipo Anogramma Link}

Figuras 40-41

Radiossimétrico, heteropolar, subtriangular a triangular em vista polar, zonas inter-angulares retas a levemente convexas. Trilete, margo desenvolvido. Exospório com projeções alongadas e robustas no pólo distal, formando figura triangular com saliência irregular central. Eixo equatorial: cerca de $65 \mu \mathrm{m}$.

Dados ecológicos: o gênero ocorre como terrícola em áreas úmidas de variados ambientes (Tryon \& Tryon 1982). No Rio Grande do Sul distribuise aproximadamente entre 20 e $900 \mathrm{~m}$ de altitude (Lorscheitter et al. 2001).

Família Blechnaceae

\section{Blechnum cf. imperiale (Fée \& Glaziou) H Chr.}

Figura 42

Bilateral, amarelado-escuro, heteropolar, oblato e elipsoidal. Elíptico em vista polar e plano-convexo em vista equatorial. Monolete com margo, exospório espesso, psilado. Robusto. Eixo polar: 70-87 $\mu$ m. Eixo equatorial maior: 94-110 $\mu \mathrm{m}$.

Dados ecológicos: espécie sub-arborescente vastamente dispersa em locais pantanosos e úmidos, junto de águas correntes no campo, mas também em locais mais secos em matas e capoeiras. No Rio Grande do Sul ocorre preferencialmente em turfeiras do Planalto (Sehnem 1968).

\section{Tipo Blechnum L.}

Figura 43

Bilateral, amarelo-claro, heteropolar, oblato e elipsoidal. Elíptico em vista polar e plano-convexo em vista equatorial. Monolete com margo, exospório psilado. Eixo polar: $36-40 \mu \mathrm{m}$. Eixo equatorial maior: 53-55 $\mu \mathrm{m}$.

O esporo do Tipo Blechnum difere do de $B$. cf. imperiale pelo menor tamanho e pela cor mais clara.

Dados ecológicos: o gênero é composto por plantas terrícolas, rupestres ou algumas vezes epifíticas. Na América tropical cresce em florestas tropicais, florestas de montanha e florestas nebulares. Também em margem de córregos e rios. Ocorre em pântanos e turfeiras, em florestas de galeria e em savanas. Algumas espécies vivem em locais alterados (Tryon \& Tryon 1982). Gênero predominante no hemisfério sul (Sehnem 1968).
Família Polypodiaceae

25. Pecluma pectinatiformis (Lindm.) M.G. Price

Figuras 44-47

Bilateral, heteropolar, oblato e elipsoidal. Elíptico em vista polar. Monolete com margo. Exospório verrucado, verrugas robustas e de tamanho uniforme, expandidas e distanciadas, de contorno regular. Eixo polar: cerca de $50 \mu \mathrm{m}$. Eixo equatorial maior: 63-70 $\mu \mathrm{m}$. Eixo equatorial menor: cerca de $40 \mu \mathrm{m}$.

Dados ecológicos: espécie epifítica, ocasionalmente epipétrica ou terrícola, em florestas. No Rio Grande do Sul encontra-se entre 30 e 1.100 $\mathrm{m}$ de altitude (Lorscheitter et al. 2005).

26. Tipo Microgramma vacciniifolia (Langsd. \& Fisch.) Copel.

Figura 48

Bilateral, heteropolar, oblato e elipsoidal. Elíptico em vista polar e plano-convexo em vista equatorial. Monolete. Exospório verrucado, verrugas robustas e irregulares, distribuídas por toda a superfície, diminuindo em direção ao pólo proximal. Eixo polar: 59-73 $\mu \mathrm{m}$. Eixo equatorial maior: $78-97 \mu \mathrm{m}$.

Dados ecológicos: a espécie é epifítica a epipétrica, em locais sombreados e abertos, desde florestas de baixas altitudes até florestas nebulares e em rochas semissombreadas. No Rio Grande do Sul é encontrada em quase todas as regiões fisiográficas (Lorscheitter et al. 2005).

\section{Tipo Polypodium L.}

Figura 49

Bilateral, heteropolar, oblato e elipsoidal. Elíptico em vista polar, plano-convexo em vista equatorial. Monolete, margo geralmente desenvolvido. Exospório verrucado, verrugas pequenas, uniformemente distribuídas. Eixo polar: 46-59 $\mu \mathrm{m}$. Eixo equatorial maior: $67-82 \mu \mathrm{m}$.

Diferencia-se do Tipo M. vacciniifolia por apresentar verrugas pequenas e uniformes por toda a superfície.

Dados ecológicos: o gênero apresenta espécies de ambientes variados. Na América tropical geralmente cresce em áreas baixas de florestas tropicais, florestas de montanha ou florestas nebulares. (Tryon \& Tryon 1982). No Rio Grande do Sul ocorre, em geral, como plantas epifíticas em matas (Lorscheitter et al. 2005). 

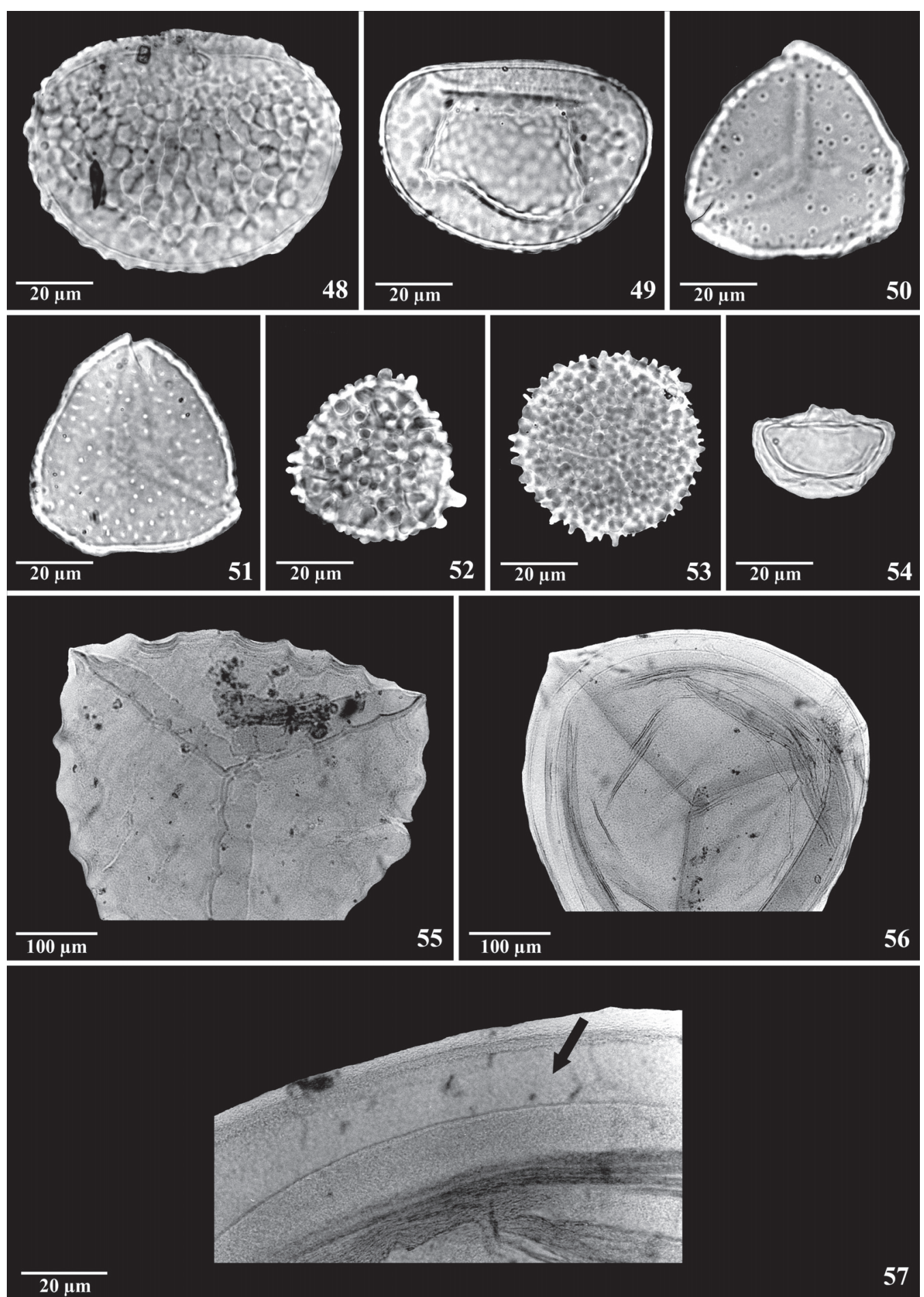

Figuras 48-57. Pteridófitos. 48. Tipo Microgramma vacciniifolia (EQ). 49. Tipo Polypodium (EQ). 50-51. Huperzia (PD): $1^{\circ}-2^{\circ} \mathrm{pl}(\mathrm{marca}$ trilete vista por transparência). 52-53. Selaginella marginata. 52. (PD), 53. (PP). 54-57. Isoëtes. 54. Micrósporo (EQ). 55-57. Megásporos. 55. (PP), reticulado, 56. (PD) psilado (marca trilete vista por transparência), 57. Exospório com lacuna (seta). ( $\mathrm{pl}=$ planos de focalização; $\mathrm{PD}=$ pólo distal $; \mathrm{PP}=$ pólo proximal; $\mathrm{EQ}=$ vista equatorial $)$.

Figures 48-57. Pteridophytes. 48. Microgramma vacciniifolia type (EQ). 49. Polypodium type (EQ). 50-51. Huperzia (PD): $1^{\circ}-2^{\circ} \mathrm{pl}(1 \mathrm{laesura}$ seen due to transparency). 52-53. Selaginella marginata. 52. (PD), 53. (PP). 54-57. Isoëtes. 54. Microspore (EQ). 55-57. Megaspores. 55. $(\mathrm{PP})$, reticulate, 56. (PD) laevigate (laesura seen due to transparency). 57. Exospore with lacune (arrow). ( $\mathrm{pl}=\mathrm{planes} ; \mathrm{PD}=\mathrm{distal}$ pole; $\mathrm{PP}=$ proximal pole; $\mathrm{EQ}=$ equatorial view). 
Classe Lycopodiopsida

Ordem Lycopodiales

Família Lycopodiaceae

\section{Huperzia Bernh.}

Figuras 50-51

Radiossimétrico, heteropolar, subtriangular em vista polar, com zonas inter-angulares levemente convexas. Trilete. Exospório foveolado no pólo distal, psilado no proximal. Perfurações regularmente distribuídas. Eixo equatorial: 53-59 $\mu \mathrm{m}$.

Dados ecológicos: gênero com a maioria das espécies epífíticas, ocorrendo dentro de matas (Lorscheitter et al. 1998).

Ordem Selaginellales

Família Selaginellaceae

29. Selaginella marginata (Humb. \& Bonpl. ex Willd.) Spring

Figuras 52-53

Radiossimétrico, heteropolar, circular a subtriangular com zonas inter-angulares convexas em vista polar. Trilete. Exospório baculado, báculas pequenas, menores no pólo proximal, densamente distribuídas. Eixo equatorial: 40-49 $\mu \mathrm{m}$.

Dados ecológicos: terrícola, em ambientes úmidos, comuns no Rio Grande do Sul (Lorscheitter et al. 1998).

Ordem Isoëtales

Família Isoëtaceae

\section{Isoëtes L.}

Micrósporo

Figura 54

Bilateral, heteropolar, oblato, hialino. Elíptico em vista polar, plano-convexo em vista equatorial. Monolete. Exospório psilado. Paraexospório psilado a escabrado, envolvendo frouxamente o grão. Eixo polar: com paraexospório: 22-37 $\mu \mathrm{m}$, sem paraexospório: 13$27 \mu \mathrm{m}$. Eixo equatorial maior: com paraexospório: 30-39 $\mu \mathrm{m}$, sem paraexospório: cerca de $30 \mu \mathrm{m}$.

Megásporo

Figuras 55-57

Radiossimétrico, heteropolar. Subtriangular a circular em vista polar, com cingulum. Trilete, freqüentemente com raios de extremidade auriculada. Reticulado a escabrado no pólo distal, psilado no proximal. Exospório externo lamelar e lacunoso, lacuna fina a espessa. Eixo equatorial: 450-500 $\mu \mathrm{m}$.

Os megásporos foram encontrados no material maior que $250 \mu \mathrm{m}$, separado durante a filtragem de cada amostra.

Dados ecológicos: gênero de plantas aquáticas, palustres ou terrícolas, geralmente submersas e/ ou anfíbias em lagos de águas rasas ou margem de lagos, poças ou córregos, solos arenosos úmidos e em turfeiras, com distribuição cosmopolita (Tryon \& Tryon 1982, Fuchs-Eckert 1986). Em banhados e águas correntes no Rio Grande do Sul e Santa Catarina (Fuchs-Eckert 1986).

Os resultados obtidos na presente pesquisa apontam vários táxons, incluindo nove fungos, cinco algas, três briófitos e 13 pteridófitos, num total de 30 palinomorfos distintos, preservados no perfil sedimentar da turfeira do Banhado Amarelo, no intervalo de tempo correspondente aos últimos 26.000 anos. A grande riqueza de material e ambientes associados indica o potencial da palinologia de sedimentos de turfeiras do Planalto do Rio Grande do Sul em oferecer excelente ferramenta para o estudo da dinâmica da vegetação dos últimos milênios. Lophosoria quadripinnata (J.F. Gmelin) Christensen é a primeira citação para o Quaternário do Sul do Brasil.

\section{Agradecimentos}

As autoras agradecem as bolsas de pesquisa concedidas e os auxílios financeiros do CNPq, fundamentais para o desenvolvimento desta pesquisa.

\section{Literatura citada}

Behling, H., Bauermann, S.G. \& Neves, P.C.P. 2001. Holocene environmental changes in São Francisco de Paula region, southern Brazil. Journal of South American Earth Sciences 14: 631-639.

Behling, H. \& Pillar, V.D.P. 2007. Late Quaternary vegetation, biodiversity and fire dynamics on the southern Brazilian highland and their implication for conservation and management of modern Araucaria forest and grassland ecosystems. Philosophical Trasactions of the Royal Society B 362: 243-251.

Behling, H., Pillar, V.D.P., Orlóci, L. \& Bauermann, S.G. 2004. Late Quaternary Araucaria forest, grassland (campos), fire and climate dynamics, studied by high resolution pollen, charcoal and multivariate analysis 
of the Cambará do Sul core in southern Brazil. Palaeogeography, Palaeoclimatology, Palaeoecology 203: 277-297.

Berglund,B.E. 1986. Handbook of Holocene Palaeoecology and Palaeohydrology. John Wiley \& Sons, New York.

Bold, H.C., Alexopoulos, C.J. \& Delevoryas, T. 1987. Morphology of plants and fungi. 5 ed. Harper \& Row Publishers, New York.

CABI - BIOSCIENCE DATABASES. 2008. The Dictionary of the Fungi, 9 ed. 2001. http: //www. speciesfungorum.org (acesso em 17.09.2008).

Ciferri, R. 1953 Atti dellIstituto Botanico della Università e Laboratorio Crittogamico, Pavia, Serie 5, 10: 218.

Christopher, R.A. 1976. Morphology and taxonomic status of Pseudoschizaea Thiergart and Frantz ex R. Potonie Emend. Micropaleontology 22: 143-150.

Erdtman, G. 1969. Handbook of Palynology. Hafner Publishing, New York.

Faegri, K. \& Iversen, J. 1989. Textbook of pollen analysis. 4 ed. John Wiley \& Sons. New York.

Fuchs-Eckert, H.P. 1986. Isoetáceas. In: R. Reitz \& R.M. Klein (eds.) Flora Ilustrada Catarinense, Herbário Barbosa Rodrigues, Itajaí. pp. 1-42.

Hooghiemstra, H. 1984. Vegetational and climatic history of the High Plain of Bogotá, Colombia: A continuous record of the last 3.5 million years. Strauss \& Cramer, Amsterdam.

Joly,A.B. 2002. Botânica: introdução à taxonomia vegetal. 13 ed. Editora Nacional, São Paulo.

Kirk, P.M., Cannon, P.F., David, J.C. \& Stalpers, J.A. 2001. Dictionary of the fungi. 9 ed. CABI Bioscience, Cambridge.

Leal, M. \& Lorscheitter, M.L. 2006. Pólen, esporos e demais palinomorfos de sedimentos holocênicos de uma floresta paludosa, Encosta Inferior do Nordeste, Rio Grande do Sul, Brasil. Iheringia, série Botânica 63: 69-100.

Leonhardt, A. \& Lorscheitter, M.L. 2007. Palinomorfos do perfil sedimentar de uma turfeira em são Francisco de Paula, Planalto Leste do Rio Grande do Sul, sul do Brasil. Revista Brasileira de Botânica 30: 47-59.

Lorscheitter, M.L. 1989. Palinologia de sedimentos quaternários do testemunho T15, Cone do Rio Grande, Atlântico Sul, Brasil. Descrições taxonômicas. Parte II. Pesquisas 22: 89-127.

Lorscheitter, M.L., Ashraf, A.R., Bueno, R.M. \& Mosbrugger, V. 1998. Pteridophyte of Rio Grande do Sul flora, Brazil. Part I. Palaeontographica 246: 1-113.

Lorscheitter, M.L., Ashraf, A.R., Windisch, P.G. \& Mosbrugger, V. 1999. Pteridophyte of Rio Grande do Sul flora, Brazil. Part II. Palaeontographica 251: 71-235.
Lorscheitter, M.L., Ashraf, A.R., Windisch, P.G. \& Mosbrugger, V. 2001. Pteridophyte of Rio Grande do Sul flora, Brazil. Part III. Palaeontographica 260: 1-165.

Lorscheitter, M.L., Ashraf, A.R., Windisch, P.G. \& Mosbrugger, V. 2005. Pteridophyte of Rio Grande do Sul flora, Brazil. Part V. Palaeontographica 270: 1-180.

Matteri, C.M. 1987. Esporas de hongos en capsulas de Sphagnum magellanicum de Tierra del Fuego. Boletín de la Associación Latinoamericana de Paleobotánica y Palinologia 10: 6.

Menéndez, G.G.H. 1962. Estudio de las Anthocerotales y Marchantiales de la Argentina. Instituto Miguel Lillo, Tucumán.

Neves, P.C.P. \& Bauermann. S.G. 2003. Catálogo palinológico de coberturas quaternárias do Estado do Rio Grande do Sul (Guaíba e Capão do Leão), Brasil. Descrições taxonômicas - Parte I: fungos, algas, palinomorfos outros e fragmentos de invertebrados. Pesquisas, Botânica 53: 121-149.

Neves, P.C.P. \& Bauermann. S.G. 2004. Catálogo palinológico de coberturas quaternárias do Estado do Rio Grande do Sul (Guaíba e Capão do Leão), Brasil. Descrições taxonômicas - Parte II: Bryophyta e Pteridophyta. Pesquisas, Botânica 55: 227-251.

Neves, P.C.P. \& Lorscheitter, M.L. 1992. Palinologia de sedimentos de uma mata tropical paludosa em Terra de Areia, Planície Costeira Norte, Rio Grande do Sul, Brasil. Descrições Taxonômicas, Parte I: fungos, algas, briófitos, pteridófitos, palinomorfos outros e fragmentos de invertebrados. Acta Geológica Leopoldensia 15: 83-114.

Punt, W., Blackmore, S., Nilsson, S. \& Thomas, A.L.E. 2007. Glossary of pollen and spore terminology. Review of Paleobotany and Palynology 143: 1-81.

Rossignol, M. 1962. Analyse pollinique de sédiments marins quaternaries em Israel. II Sédiments Pleistocenes. Pollen et Spores 4: 121-148.

Roth, L. \& Lorscheitter, M.L. 1993. Palynology of a bog in Parque Nacional de Aparados da Serra, East Plateau of Rio Grande do Sul, Brazil. Quaternary of South America and Antarctic Peninsula 8: 39-69.

Roth, L. \& Lorscheitter, M.L. 2008. Palinomorfos de um perfil sedimentar em uma turfeira do Parque Nacional dos Aparados da Serra, leste do Planalto do Rio Grande do Sul, Brasil. Iheringia, série Botânica 63: 69-100.

Round,F.E. 1973. The Biology of the Algae. 2 ed. Edward Arnold, London.

Scherer, C. \& Lorscheitter, M.L. 2008. Palinomorfos de fungos e criptógamas em sedimentos quaternários de duas matas com Araucária, Planalto leste do Rio Grande do Sul, Brasil. Acta Botanica Brasilica 22: 131-144. 
Schultz, A.R.H. 1980. Introdução à botânica sistemática. 5 ed., v.1.Editora da Universidade Federal do Rio Grande do Sul, Porto Alegre.

Schüßler, A., Schwarzott, D. \& Walker, C. 2001. A new fungal phylum, the Glomeromycota: phylogeny and evolution. Mycological Research 105: 1413-1421.

Sehnem, A. 1968. Blecnáceas. In: R. Reitz (ed.). Flora Ilustrada Catarinense. Herbário Barbosa Rodrigues, Itajaí.

Smith, G.M. 1987. Botanica criptogamica. 4 ed., v.1. Fundação Calouste Gulbenkian, Lisboa.
Tryon, R.M. \& Tryon, A.F. 1982. Ferns and allied plants. Springer-Verlag, New York.

Van Geel, B. 1978. A paleoecological study of Holocene peat bog section in Germany and the Netherlands. Review of Palaeobotany and Palynology 25: 1-120.

Van Geel, B. \& Van Der Hammen, T. 1977.Zygnemataceae in Quaternary Colombian sediments. Review of Palaeobotany and Palynology 25: 377-392.

Yano, O., Pirani, J.R. \& Santos, D.P. 1985. O gênero Sphagnum (Bryopsida) nas regiões Sul e Sudeste do Brasil. Revista Brasileira de Botânica 8: 55-80. 